\title{
Aa. Vv., Joseph de Maistre and the legacy of Enlightenment
}

Esther Pinon

\section{(2) OpenEdition}

\section{Journals}

Édition électronique

URL : https://journals.openedition.org/studifrancesi/4721

DOI : 10.4000/studifrancesi.4721

ISSN : 2427-5856

Éditeur

Rosenberg \& Sellier

\section{Édition imprimée}

Date de publication : 1 avril 2012

Pagination : 159-160

ISSN : 0039-2944

\section{Référence électronique}

Esther Pinon, «Aa. Vv., Joseph de Maistre and the legacy of Enlightenment », Studi Francesi [En ligne], 166 ( | LVI) | 2012, mis en ligne le 30 novembre 2015, consulté le 18 novembre 2021. URL : http:// journals.openedition.org/studifrancesi/4721 ; DOI : https://doi.org/10.4000/studifrancesi.4721

Ce document a été généré automatiquement le 18 novembre 2021.

\section{(c) $(1) \&$}

Studi Francesi è distribuita con Licenza Creative Commons Attribuzione - Non commerciale - Non opere derivate 4.0 Internazionale. 


\title{
Aa. Vv., Joseph de Maistre and the legacy of Enlightenment
}

\author{
Esther Pinon
}

\section{RÉFÉRENCE}

AA. VV., Joseph de Maistre and the legacy of Enlightenment, Oxford, Voltaire foundation, 2011 («SVEC»: 1), pp. 254.

1 Les articles réunis par Carolina ARMENTEROS et Richard A. LEBRUN dans le volume intitulé Joseph de Maistre and the legacy of Enlightenment constituent les actes du Cinquième Colloque International sur Joseph de Maistre, qui s'est déroulé les 4 et 5 décembre 2008 à Cambridge, au Jesus College. Ils proposent une relecture de l'œuvre de Maistre, et particulièrement de son rapport aux Lumières: la figure du conservateur pessimiste et obscurantiste, volontiers tenu pour un précurseur du fascisme depuis les travaux d'Isaiah Berlin, y est fortement remise en question et nuancée.

2 Cette quête de la nuance est d'ailleurs annoncée dès l'introduction (pp.1-16), dans laquelle les deux éditeurs posent les enjeux du sujet en le replaçant dans l'histoire des études maistriennes. En suggérant que Maistre pourrait être considéré autant comme un représentant du mouvement des Lumières que comme l'un de ses plus ardents adversaires, ils rappellent l'irréductible complexité (et donc la richesse) de l'auteur étudié, mais aussi du contexte historique et philosophique du XVIII siècle et du XIX ${ }^{\mathrm{e}}$ siècle naissant.

Le volume, qui mêle les approches littéraires, philosophiques et théologiques sans jamais perdre de son unité, est organisé en trois temps. Sont tout d'abord étudiées quelques - unes des grandes thématiques de l'œuvre de Maistre qui révèlent à quel point sa pensée se construit à la fois contre et avec l'héritage des Lumières. Dans cette perspective, Darrin M. MCMAHON (The genius of Maistre, pp. 19-30) et Joseph EATON ("This babe-in-arms": Joseph de Maistre's critique of America, pp. 31-43) étudient respectivement son approche du «génie» et sa critique de la démocratie américaine, tandis que Jean- 
Yves PRANCHÈRE (The negative of the Enlightenment, the positive of order and the impossible positivity of history, pp.45-64) se penche sur son rapport à l'Histoire en montrant combien ce rapport, conditionné par le besoin de retrouver un ordre bouleversé par la Révolution, informe son opposition à la philosophie des Lumières.

La deuxième partie de l'ouvrage est consacrée à une mise en perspective de l'œuvre de Maistre par sa confrontation avec celle d'autres auteurs, ses prédécesseurs ou ses contemporains: Philippe BARTHELET (The Cambridge Platonists mirrored by Joseph de Maistre, pp. 67-77) montre sa dette à l'égard de l'école des Platoniciens de Cambridge et Carolina ARMENTEROS (Maistre's Rousseau, pp. 79-103), celle qui le lie à Rousseau - elle révèle entre les deux penseurs un lien étroit, mais contrasté, selon que Maistre voit en Rousseau un critique de la révélation, un théoricien de la conscience et de la liberté, ou un pédagogue. Enfin, Yannis CONSTANTINIDÈs (Two great enemies of the Enlightenment: Joseph de Maistre and Schopenhauer, pp. 105-122), dans un article plus surprenant peut-être, mais non moins fécond, dresse un parallèle entre Maistre et Schopenhauer. Il ne s'agit plus cette fois d'influences, même indirectes, mais dans leurs différences autant que dans leurs points communs (une vision tragique de l'existence, qui n'exclut pourtant pas la perspective d'un salut), les œuvres des deux auteurs s'éclairent mutuellement.

Quant aux quatre derniers articles, ils se concentrent sur les questions plus spécifiquement théologiques que soulèvent les écrits de Maistre, toujours en les mettant en regard avec la pensée des Lumières. Douglas HEDLEY (Enigmatic images of an invisible world: sacrifice, suffering and theodicy in Joseph de Maistre, pp. 125-146) examine les implications du motif du sacrifice sur lequel repose une théodicée qui, selon lui, révèle en Maistre moins un opposant réactionnaire des Lumières qu'un auteur de son temps, profondément romantique. Émile PERREAU-SAUSSINE (Why Maistre became Ultramontane, pp.147-159) revient sur les raisons qui motivent l'ultramontanisme de Maistre et Aimee E. BARBEAU (The Savoyard philosopher: deist or Neoplatonist?, pp. 161-189) retrouve dans la pensée religieuse de l'auteur les influences combinées du déisme et du néoplatonisme chrétien. Quant à Élcio VERçOSA FILHO (The pedagogical nature of Maistre's thought, pp.191-219), il propose de voir dans la démarche pédagogique de Maistre (particulièrement perceptible dans Les Soirées de Saint-Pétersbourg) un principe unificateur de l'œuvre, et une forme paradoxale de lien avec le mouvement des Lumières: à sa manière, et souvent contre les Lumières, Maistre cherche lui aussi à éclairer son lecteur.

6 Au terme de son parcours, c'est en somme un Joseph de Maistre lui aussi «éclairé» que l'on découvre, tout d'abord au sens où il s'avère, en bien des aspects, nourri de la pensée du XVIII ${ }^{e}$ siècle, mais aussi parce que l'ensemble des articles constitue en quelque sorte ce que l'on pourrait nommer un «Éclaircissement sur Joseph de Maistre». L'auteur se trouve replacé, non seulement dans le contexte qui est immédiatement le sien, mais également dans des perspectives plus larges qui le montrent d'une part influencé par la culture riche et diverse des siècles passés - on songe au néoplatonisme chrétien, et notamment à Origène -, et de l'autre annonciateur du siècle qu'il a précédé: à plusieurs reprises, les contributeurs suggèrent qu'il a sans doute moins préfiguré les totalitarismes du $\mathrm{xx}^{\mathrm{e}}$ siècle qu'il n'en a pressenti les excès et la violence. 